\title{
Transferir para documentar el patrimonio cultural. Curso superior de métodos y técnicas para la documentación e información del patrimonio cultural
}

El pasado mes de julio finalizaba la primera edición del Curso superior de métodos y técnicas para la documentación e información del patrimonio cultural. Dirigido, coordinado y gestionado por el Instituto Andaluz del Patrimonio Histórico, comenzó en octubre de 2014, con una duración de 240 horas. Con este curso, en la modalidad de teleformación, el IAPH apuesta por un nuevo modelo formativo que confía se consolide y responda a las exigencias de los y las profesionales del patrimonio cultural en el nuevo y difícil contexto socio-económico que vivimos. Satisfechos con los resultados, se está trabajando ya en la II edición (otoño de 2016). Ofrecemos aquí un balance del curso con algunos comentarios que nos ha hecho llegar el alumnado.

\section{Redacción IAPH}

URL de la contribución <www.iaph.es/revistaph/index.php/revistaph/article/view/3683>

Desde 2013 el IAPH trabaja en nuevas fórmulas para materializar la transferencia del conocimiento en las materias que son de su competencia. Ya no se trata de transmitir una técnica o de presentar un proyecto, sino de abrir las puertas a quienes trabajan en las distintas vertientes del patrimonio cultural mostrando su metodología, fruto del trabajo de más de 25 años de trayectoria de la institución. Esta transferencia podía realizarse a través de distintos medios pero, para llegar de verdad a transmitir una forma de hacer, de pensar, se apostó por gestionar el tiempo a largo plazo; es decir, la formación superior con cursos de larga duración para garantizar el aprendizaje de un método, en los que el alumnado pudiera desarrollar un proyecto real. La primera experiencia en este sentido fue el "Curso superior de intervención en bienes muebles: el proyecto de conservación", cuya primera edición tuvo lugar los años 2013-2014. Una vez finalizado, comenzaron las primeras reuniones para dar forma al segundo curso, esta vez en el ámbito de la documentación e información de bienes culturales.

Tanto en el diseño como en el programa de este curso se ha querido trasladar el espíritu interdisciplinar del IAPH. Para romper las barreras geográficas se planteó un curso en formato de teleformación. Era la primera vez que nos enfrentábamos a un modelo de larga duración en línea, pero las preinscripciones recibidas (169), en una época en la que peligran las profesiones vinculadas al patrimonio, nos animaron a seguir:

"El formato del curso online, bastante extendido actualmente, tiene como ventajas fundamentales las siguientes: en primer lugar, la accesibilidad a la realización del curso puesto que de ser presencial no hubiera sido posible y, en segundo lugar, su flexibilidad, ya que este formato permite compatibilizar las actividades profesionales y personales"

Jorge Molinero Sánchez, arquitecto, Granada (España)

"Es mi primer curso en línea y ha sido muy gratificante y fácil de manejar. He podido atender mis obliga-

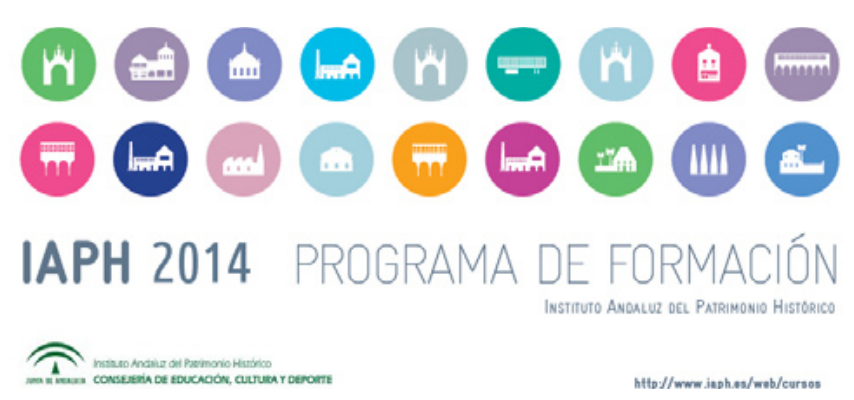


ciones laborales y familiares y al mismo tiempo aprender, contando con la paciencia de todo el profesorado"

Leticia Ivonne del Río Hernández, historiadora del arte, Zacatecas (México)

El reto y la responsabilidad para con el alumnado han sido muy importantes para el personal técnico del IAPH: fueron admitidos 32 profesionales, procedentes de 11 comunidades autónomas españolas, así como del Estado de Zacatecas en México y el Departamento de Santander en Colombia. Casi toda la plantilla del Centro de Documentación y Estudios y del Departamento de Formación, así como dos profesionales con experiencia en las materias impartidas (un total de 25 personas), han participado desarrollando tutorías, elaborando materiales, gestionado la plataforma, participando en foros o actividades o resolviendo dudas:

"Principalmente quiero agradecer la labor de todos los profesores que han participado en los distintos módulos del curso, y cuya experiencia, disponibilidad y profesionalidad nos han sido de gran ayuda para ir superando las distintas materias abordadas y poder realizar los trabajos recomendados"

Rosario Pérez Lorente, historiadora del arte, Granada España)

"El profesorado de altísimo nivel y especialización nos ha facilitado la documentación necesaria para el desarrollo de cada uno de los módulos, contestando a las dudas de un modo efectivo y proponiendo temas y cuestiones que han provocado que los foros sean dinámicos"

Jorge Molinero Sánchez, arquitecto, Granada (España)

Compartir espacios comunes, como los foros, ha dado la oportunidad de valorar patrimonios, perspectivas y problemáticas muy diversas. Se ha trabajado el patrimonio, partiendo de su definición y construcción social, su contextualización en la sociedad de la información en la que nos encontramos, hasta la materialidad del bien mueble, inmueble, material e inmaterial, tocando patri- monios emergentes como el patrimonio subacuático o el paisaje cultural, y algunos aspectos relacionados con su integración en instrumentos de planificación territorial. El conjunto de las materias ofrece una visión global y multidisciplinar del patrimonio cultural desde las perspectivas que el IAPH propone para su utilización como herramientas de información, integrando también normativa y legislación, conceptos básicos sobre las tecnologías de la información geográfica o recomendaciones para la documentación cartográfica, gráfica y textual del patrimonio, entre otros temas:

"Desde el punto de vista de la documentación y catalogación del patrimonio cultural considero que el curso es muy completo ya que abarca un amplio ámbito de las tipologías patrimoniales, tanto las tradicionales como los patrimonios emergentes; así como módulos expertos sobre procedimientos para la documentación bibliográfica y documental, fotográfica y digitalización, sistemas de información geográfica y el tratamiento de la información patrimonial en la sociedad de la información"

Itziar Azua Brea, historiadora y gestora cultural, Bizkaia (España)

"Por el entorno en el que vivo, el mundo subacuático, en principio, escapaba de mis curiosidades, pero con la lectura y la tarea pude comprender aspectos que me han cambiado la percepción del patrimonio cultural en el fondo de los mares y ha puesto de manifiesto nuestra historia común. El territorio, pensado como paisaje cultural me ha brindado alcances nuevos"

Leticia Ivonne del Río Hernández, historiadora del arte, Zacatecas (México)

"El temario es completo y extenso, abarcando materias algunas de las cuales me han sorprendido por ser nuevas para mí, y todas con un contenido teóricopráctico suficiente para adquirir los conocimientos que nos permitan posteriormente continuar profundizando en aquellos aspectos que nos interesen"

Jorge Molinero Sánchez, arquitecto, Granada (España) 


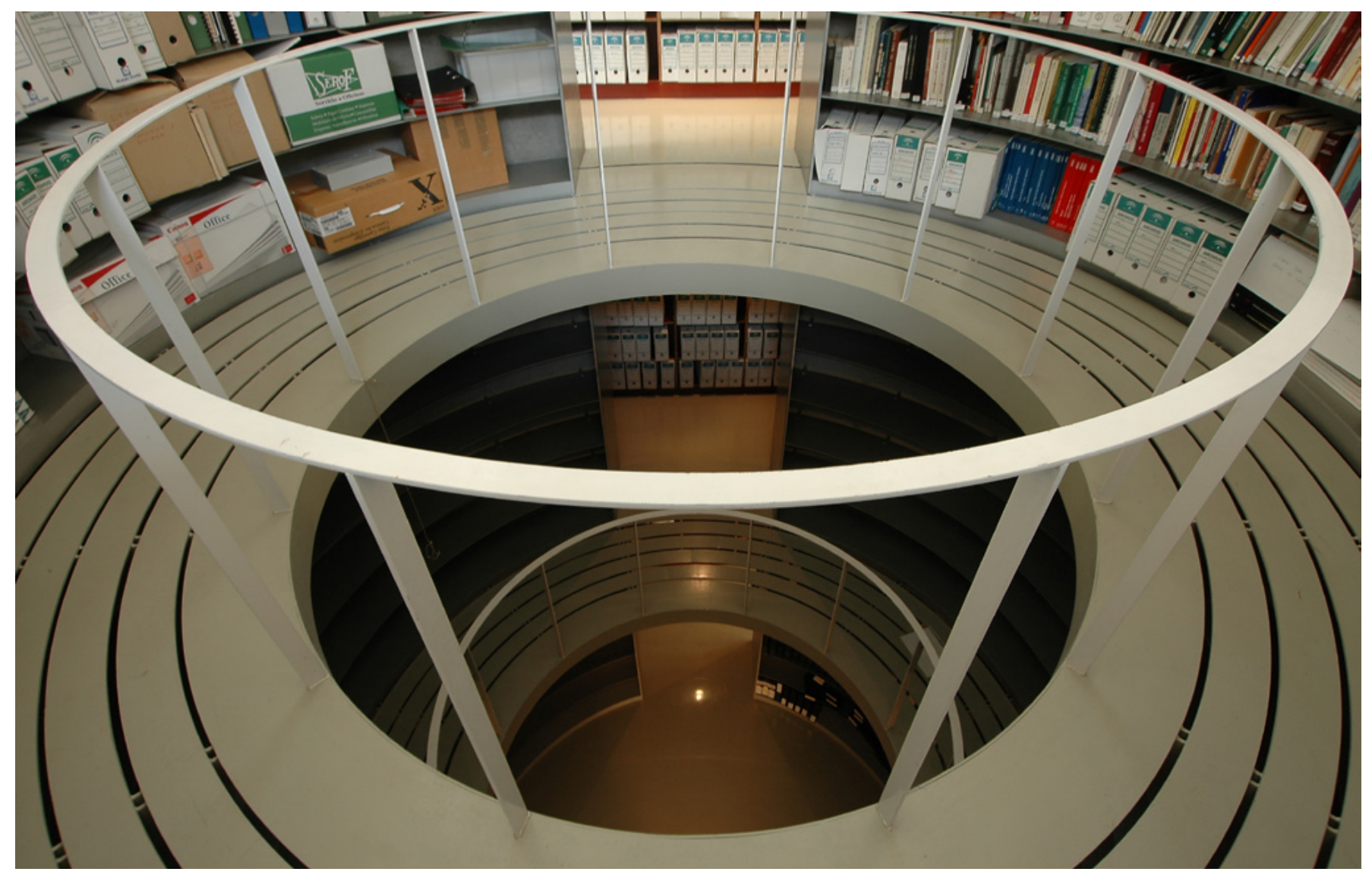

Interior del archivo del IAPH | foto Fondo Gráfico IAPH

Para la formación en línea se ha utilizado una plataforma, Moodle, como escenario de enseñanza-aprendizaje, empleando contenidos elaborados de forma didáctica, con esquemas, resúmenes y ejemplificaciones para su mayor comprensión y claridad. El curso tenía un doble enfoque: una parte teórica, en la que el alumnado debía leer los contenidos de los módulos, participar en los foros de debate, realizar las actividades y cuestionarios; y otra parte práctica, con la realización de un proyecto final individual tutorizado por el profesorado del curso:

"La temática de los módulos ha sido interesantísima, la documentación muy apropiada en todo momento y el profesorado con una disponibilidad absoluta y atento a resolver las dudas. Creo que una de las actividades más enriquecedoras han sido los debates, ya que compartir la experiencia de profesores y demás compañeros de diferentes ámbi- tos y lugares es una de las fuentes más valiosas de aprendizaje"

Rosario Pérez Lorente, historiadora del arte, Granada (España)

"En cuanto a la idoneidad y adecuación metodologica, me ha parecido correcta en general: la metodología sigue unos criterios generales de lecturas y ejercicios obligatorios y bibliografía complementaria, con calidad y nivel de actualización que varía según los módulos (hay módulos que me han gustado especialmente: $1^{\circ}$, $\left.2^{\circ}, 7^{\circ}, 8^{\circ}, 10^{\circ} \ldots\right)$, y con un enfoque experto general que, en algún caso, como el relativo a la documentación fotógrafica, me ha parecido incluso excesivo en un curso de carácter amplio. En general, el sistema de evaluación a base de ejercicios, trabajos y sobre todo las exposiciones en el foro me parecen bastante más 
adecuados para la asimilación y desarrollo de los contenidos que los test sobre las lecturas obligatorias"

Itziar Azua Brea, historiadora y gestora cultural, Bizkaia (España)

"Los trabajos propuestos en aplicación del contenido teórico han sido adecuados para cumplir los objetivos de cada tema, únicamente indicar que algún módulo se ha quedado corto en tiempo para su desarrollo y otros quizás podían haber tenido menos dedicación"

Jorge Molinero Sánchez, arquitecto, Granada (España)

$Y$ todo ello para formar profesionales que desde su entorno más cercano puedan incidir en el conocimiento de su patrimonio, ya que dominar las técnicas de documentación e información proporciona herramientas para la protección, gestión y difusión del patrimonio cultural. Un gran esfuerzo que esperamos sirva para contribuir a formar y reforzar el papel de quienes trabajan en un sector que resiste en un contexto económico especialmente difícil y tan frágil y necesario como son los hilos que nos ligan a un grupo, una comunidad o una identidad, al conocimiento de nuestro pasado y a una apuesta de futuro asentada sobre nuevas bases, más sostenibles y respetuosas con el legado común:

"Personalmente el curso ha cumplido ampliamente con mis expectativas de reciclaje y actualización"

Itziar Azua Brea, historiadora y gestora cultural, Bizkaia (España)

"El curso me aportó sistematicidad, disciplina y rigor para abordar el estudio del patrimonio cultural dignificando su importancia; me ayudó a sentar las bases para procesar información que tenía dispersa sobre mi proyecto de registrar la cultura en torno a las parroquias, es decir, el patrimonio cultural de las comunidades, urbanas y rurales, de Zacatecas"

Leticia Ivonne del Río Hernández, historiadora del arte, Zacatecas (México)
"Creo que el curso da una visión muy clara de las distintas problemáticas existentes en el ámbito del patrimonio, pero también de la cantidad de profesionales especializados que trabajan y luchan para conseguir que este legado, que es de todos, llegue en las mejores condiciones a las futuras generaciones"

Rosario Pérez Lorente, historiadora del arte, Granada (España)

Satisfechos ${ }^{1}$ con los resultados, confiamos en seguir organizando futuras ediciones aprendiendo de la experiencia y con el deseo de mejorar, de ahí la importancia que le damos a la opinión de nuestro alumnado, al que agradecemos la confianza depositada en nosotros:

"Como aspectos a mejorar indicar que el trasvase de información con los compañeros solo se ha realizado a nivel de foros, echado en falta una mayor interactividad con el resto del alumnado que quizás se podía haber solventado con la realización de algún trabajo conjunto que nos hubiera permitido trabajar profesionales de diferentes campos. También hubiera sido de interés conocer al menos los trabajos más destacados de cada uno de temas desarrollados ya que solo conozco el resultado de mi trabajo no teniendo por lo tanto ningún punto de referencia de los realizados por el resto de los compañeros, tanto en la temática escogida como en el enfoque"

Jorge Molinero Sánchez, arquitecto, Granada (España)

\section{NOTA}

1. La valoración global por parte del alumnado del curso es de 9. De los 32 alumnos, han superado satisfactoriamente el curso completo 26 . 